\title{
Road tunnel safety rules in Italy: the tunnel country
}

\author{
M. Guarascio, M. Lombardi, G. Rossi \& G. Sciarra \\ Safety Engineering "Sapienza”, Rome University, Italy
}

\begin{abstract}
A case study is presented illustrating the application of the Quantitative Probabilistic Risk Analysis Methodology requested in Italy for the tunnels of the motorway road network.
\end{abstract}

Keywords: Quantitative Probabilistic Risk Analysis.

\section{Introduction}

Italy in the framework of both the rail and road network of the European Union holds significantly more than half of the overall length to be run in tunnels. That's why Italy can be considered the veritable "Tunnel Country".

Nowadays the European and Italian authorities are very active and productive as far as tunnel safety rules are considered.

Expert communities have perceived the Authorities concern with respect to the tunnel safety conditions. See for instance the European Directive [54/2004/CE] "on minimum safety requirements for tunnels in the transEuropean road network" as well as the two Italian governmental acts [D.M. 25.10.2005] "Rail Tunnel safety" and [D. Lgs. 05.06.2006] "Actuation of the D.54/2004/CE".

The aim of all the above acts is to upgrade the expected level of safety in tunnels.

The expectation of a higher level of safety in clearly declared in the Italian acts, both for rail and road cases, where are indicated in quantitative form the values to be achieved as well as criteria and procedure for demonstrating the above results.

The achievement of the requested level of safety only occurs when in a specific tunnel with given traffic, length and design there exist an adequate number of safety requirements operating at an appropriate level of performance. 
These requirements consist of infrastructural measures, like the emergency exits, or technological subsystems like ventilation and smoke control, lighting, monitoring and communication.

The rules of course take into account the specific features and hazard conditions of the rail and road environments.

In the rail world the maximum number of people involved in a fire tunnel accident could be of the order of 1000 whereas in the road case it is of 100 .

The emergency exits offer different conditions of exodus and consequently different times for the evacuation from the fire and smoke zones. In the case of road tunnels in Italy emergency exits should be compulsory located at a minimum $500 \mathrm{~m}$ distance apart.

The fire size, in terms of Heath Release Rate (HRR), megawatts (MW), temperature and smoke flow along the time in the case of rail tunnels has been identified with the reference value of $10 \mathrm{MW}$, whereas in the road case the values should range from $15 \mathrm{MW}$ to $150 \mathrm{MW}$ according to the specific traffic and transport conditions.

As far as the air and smoke flow control in the road tunnels ventilation and air control systems should be provided with a performance level increasing significantly when the length and traffic increase as well.

\section{Risk analysis for road tunnels: Italian rules}

Figure 1 shows ten types of tunnels whose reference values (European Directive 2004/54/EC / D. Lgs. 05.06.2006 “Actuation of the D.54/2004/CE”, Italian Act) for the safety parameters length (L) and traffic volume (A.A.D.T. - Annual Average Daily Traffic) comply with ten specific sets of minimum safety requirements.

Two examples of safety requirements groups are given in Figure 2.

The European Directive does not explicitly fix any upper limit for the safety parameters as length (L) and traffic volume (AADT).

\begin{tabular}{|c|c|c|c|c|c|}
\hline Unidirectional tunnel & $500<L<1000$ & $L>1000$ & $500<L<1000$ & $1000<L<3000$ & L>3000 \\
\hline $\mathrm{T}<2000 \mathrm{v} / \mathrm{l} \cdot \mathrm{d}$ & $\mathbf{I}$ & II & & & \\
\hline $\mathrm{T}>2000 \mathrm{v} / \mathrm{l} \cdot \mathrm{d}$ & & & III & v & $\mathbf{v}$ \\
\hline Bidirectional tunnel & $500<L<1000$ & $L>1000$ & $500<L<1000$ & $1000<L<3000$ & $L>3000$ \\
\hline $\mathrm{T}<2000 \mathrm{v} / \mathrm{l} \cdot \mathrm{d}$ & VI & VII & & & \\
\hline $2000<\mathrm{T}<10000 \mathrm{v} / \mathrm{l} \cdot \mathrm{d}$ & & & VIII & IX & $\mathbf{x}$ \\
\hline
\end{tabular}

Figure 1: Types of tunnel. 
IV Group $\left\{\begin{array}{l}1000<\mathrm{L}<3000 \\ \mathrm{~T}>2000 \mathrm{v} / \mathrm{ld}\end{array}\right.$

- Walkways

- Slope $<5 \%$

- Emergency exits $\leq 500 \mathrm{~m}$

- Lay-bys every $1000 \mathrm{~m}$

- Access for emergency services

- By pass outside the portal

- Drainage (ADR)

- Fire resistance and fire reaction of structures and materials

Walkways

$$
\text { V Group }\left\{\begin{array}{l}
\mathrm{L}>3000, \\
\mathrm{~T}>2000 \mathrm{v} / \mathrm{ld}
\end{array}\right.
$$

- Slope $<5 \%$

- Emergency exits $\leq 500 \mathrm{~m}$

- Lay-bys every 1000 m

- Acess for emergency services

- By pass outside the portal

- Drainage (ADR)

- Fire resistance and fire reaction of structures and materials
- Ordinary -Emergency lighting system

- Emergency ways lighting system

- Ventilaiton system

- Emergency station

- Water supply

- CCTV

- Incident and fire detection systems

- Traffic lights

- Radio communications for users (FM) and emergency services

- Loudspeaker system for shelters

- Power supply

- Fire resistance and fire reaction of devices

- Ordinary -Emergency lighting system

- Emergency ways lighting system

- Ventilaiton system

- Requirements for semi-transverse ventilation systems

- Emergency station

- Water supply

- Control Center

- CCTV

- Incident and fire detection systems

- Traffic lights

- Radio communications for users (FM) and emergency services

- Loudspeaker system for shelters

- Power supply

- Fire resistance and fire reaction of devices

Figure 2: $\quad$ Safety requirements groups IV, V.

An analysis of the rate of road accidents versus traffic volume, carried out on the basis of data relative to the Italian motorway network, shows that the regression curve presents an abrupt change in slope:

- for unidirectional tunnels the critical value of the traffic volume is 15000 vehicles/lane day.

- for bidirectional tunnels the traffic volume value of 10000 vehicles/lane day.

A similar analysis of the casualty and fatality rate due to fires in the tunnel versus tunnel length, carried out on the basis of data contained in the PIARC document (1999), and updated to 2005, has led to the identification of the following reference values: 
- for unidirectional tunnels the safety parameter limit value for length (L) is $5000 \mathrm{~m}$;

- for bidirectional tunnels the safety parameter limit value for length (L) is $3000 \mathrm{~m}$.

In the framework of a performance-based approach, the design of transport systems (IEC Regulation $n^{\circ}$ 61508) attributes specific performance functions to the safety subsystems (i.e. D. Lgs. "Road tunnel Safety"). Moreover, each safety subsystem is characterized by a specific level of integrity expressed in terms of reliability.

The above observations suggested the introduction of the following auxiliary concepts.

Virtual tunnel: a tunnel that wholly complies with the provisions of the European Directive in terms of both safety parameters and minimum requirements and for which the safety subsystems that implement the minimum requirements are characterized by ideal reliability and efficiency.

Theoretical tunnel: an existing tunnel, or its upgrading design, or a final design of the new tunnel, where not all the safety requirements are adopted, but it behaves according to ideal reliability and efficiency.

Actual tunnel: an existing tunnel, or its upgrading design, or a final design of the new tunnel, where not all the safety requirements are adopted but it behaves according to actual reliability and efficiency as per best practices.

The developed risk analysis procedure adopts the F-N plane for representing risk.

The straight line tangent envelope to the Back Cumulated Distributions (B.C.D.) derived from the Event Tree Analyses associated with virtual tunnels defines a reference limit condition on the risk representation plane.

According to the risk analysis presented below, the virtual tunnels are used as references in determining the risk level of actual tunnels.

The presented risk analysis procedure assumes the performance-based design of the structure as the characterizing feature of the tunnel safety design.

\section{Steps of the quantitative probabilistic risk analysis methodology}

The essential steps in the proposed Quantitative Probabilistic Risk Analysis procedure are:

- Characterization of the tunnel structure: Structural measures, systems measures

- Identification of the sources of hazard and corresponding incident rate: Passenger cars, coaches, heavy goods vehicles 
- Identification of potential hazards: Mechanical-thermal, chemical-thermal

- Characterization of Hazard Scenarios: Probability of triggering events, energetic characterization of sources, event tree analysis (ETA)

- Quantification and zoning of the hazard flow in the tunnel: Field of airflow, temperature, concentration of combustion products (models for mass transport and energy), size of the lethal hazard zones (effective fractional dose)

- Quantification of the damage: Exposed population (models of vehicle queues), fatalities (models for the evacuation process)

- Risk estimation and risk representation: Damage Expected Values, Back Cumulated Distributions (B.C.D.)

- $\quad$ Risk evaluation: Comparison of Back Cumulated Distributions (B.C.D.) to predefined reference terms and acceptability criteria

\section{Event Tree Analysis}

The Event Tree represents the risk estimation tool adopted in the proposed procedure. It includes the impact that the safety subsystems, expressed in terms of dependability (reliability and efficiency), have on the evolution of the hazard flow in the tunnel system along an emergency scenario $(\mathrm{Sj})$.

The issues of the Event Tree Analysis (see art. "Risk Analysis and acceptability criteria" $[1,5]$ ), repeated for a representative complete group of Initiating Events and developed up to the severity of consequences events evaluation, are used to calculate the various Risk Indicators.

As far as the fire hazard is considered in the case of the motorway network in Italy, according to the average traffic conditions it seems appropriate to adopt as independent initiating events fire accidents with thermal HRR ranging from 15 to 150 according to the different classes of vehicles and transported goods.

A study must be able to explain the effectiveness of the safety requirements on the risk level reduction when different significant values of the safety parameters length and traffic are assumed, for each value of fire hazard in terms of H.RR.

The most appropriate risk indicator in the above case to be used is the expected value of the number of fatalities $\mathrm{N}$ conditioned by the occurrence of a given Initiating Event $\left(\mathrm{HRR}_{\mathrm{i}}=15, \ldots, 150 \mathrm{MW}\right)$ and by the existence of the requested safety requirements offering the effective performance.

In the example below the Test Tunnels corresponding to the extreme values of the $\mathrm{L}$ and $\mathrm{T}$ defining the groups of safety requirements have been simulated by using a 3D CFD code and Exodus simulator considering the maximum crowded traffic condition (150 people in fire and smoke influence area). 


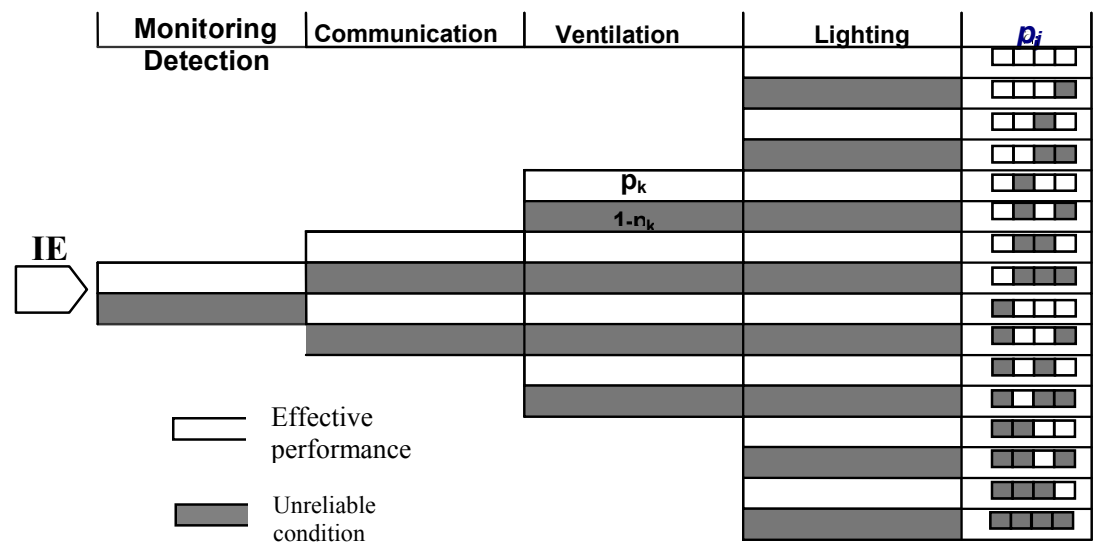

$\boldsymbol{p}_{\boldsymbol{k}}$ dependability of $\mathrm{k}^{\text {th }}$ safety subsystem

Figure 3: Event Tree Analysis.
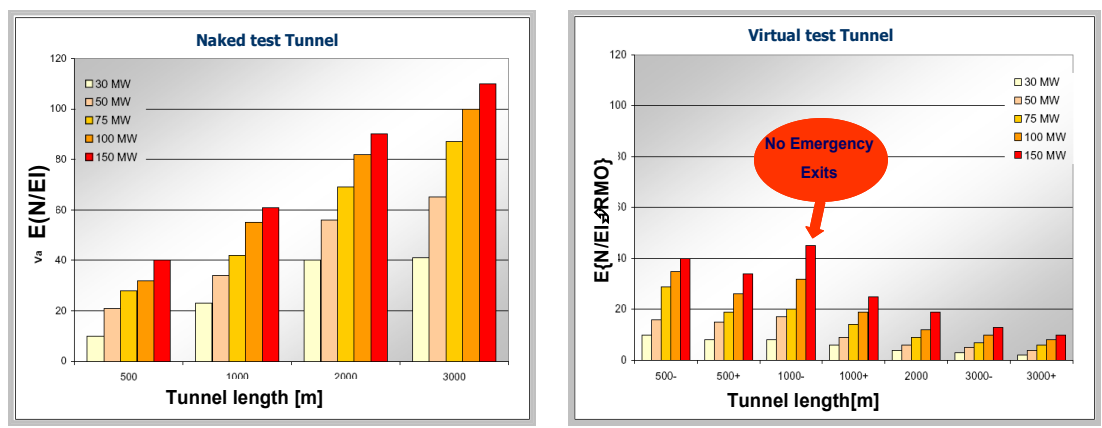

Figure 4: Left- Conditioned Expected Value of N (no Safety Requirements); Right- Conditioned Expected Value of N (Safety Requirements).

\section{Case study}

The presented case study concerns [4]:

- the application of the proposed risk analysis procedure to types $\mathrm{V}$ and $\mathrm{X}$ of virtual test tunnels in order to identify on the Plane F-N, a limit condition defined as the straight line tangent envelope to the back cumulated distributions associated with virtual test tunnels;

- the application of the proposed risk analysis procedure to three existing tunnels of the Italian road network that present various deficits with regard to the minimum safety requirements. 
Tables $1-5$ give the characterising values for virtual test tunnels types $\mathrm{V}$ and $\mathrm{X}$.

Table 1.

\begin{tabular}{ll}
\hline Name & TEST Tunnel Type V \\
Tunnel System & Unidirectional \\
Tunnel Length [m] & 5000 \\
Lanes & 2 \\
Traffic (annual average daily & 15000 \\
traffic-v/d. 1.) & \\
Heavy goods vehicles [\%] & 15 \\
Emergency Exits Distance [m] & 500 \\
Minimum Requirements Deficit & - \\
\hline
\end{tabular}

Table 2.

\begin{tabular}{ll}
\hline Name & TEST Tunnel Type X \\
Tunnel System & Bidirectional \\
Tunnel Length [m] & 3000 \\
Lanes & 1 \\
Traffic (annual average daily & 10000 \\
traffic-v/d. 1.) & \\
Heavy goods vehicles [\%] & 15 \\
Emergency Exits Distance [m] & 500 \\
Minimum Requirements Deficit & - \\
\hline
\end{tabular}

Table 3.

\begin{tabular}{ll}
\hline Name & Autostrade \\
Tunnel System & Unidirectional \\
Tunnel Length [m] & 3200 \\
Lanes & 2 \\
Traffic (annual average daily & 5500 \\
traffic-v/l·d) & \\
Heavy goods vehicles [\%] & 10 \\
Emergency Exits Distance [m] & 700 \\
Minimum Requirements Deficit & 1-Emergency Exits \\
& Distances. \\
& 2- Water supply. \\
& 3-Drainages. \\
\hline
\end{tabular}

Table 4.

\begin{tabular}{ll}
\hline Name & ANAS 1 \\
Tunnel System & Unidirectional \\
Tunnel Length [m] & 3200 \\
Lanes & 2 \\
Traffic (annual average daily & 12000 \\
traffic-v/l·d) & \\
Heavy goods vehicles [\%] & 10 \\
Emergency Exits Distance [m] & 450 \\
Minimum Requirements Deficit & - \\
\hline
\end{tabular}


Table 5.

\begin{tabular}{ll}
\hline Name & ANAS 2 \\
Tunnel System & Bidirectional \\
Tunnel Length [m] & 2400 \\
Lanes & 1 \\
Traffic (annual average daily & 10000 \\
traffic-v/l·d) & \\
Heavy goods vehicles [\%] & 10 \\
Emergency Exits Distance [m] & $800-1200$ \\
Minimum Requirements Deficit & $1-$ Emergency Exits Distances. \\
\hline
\end{tabular}

Figure 5 shows the Back Cumulated Distributions (B.C.D.) for virtual tunnels of types $\mathrm{V}$ and $\mathrm{X}$.

The straight line shown in Figure 5 represents the envelope that is tangent to the Back Cumulated Distributions (B.C.D.) associated with virtual tunnels, and it defines a reference limit condition.

Figures 6-8 show the B.C.D. for three existing tunnels of the Italian road network.

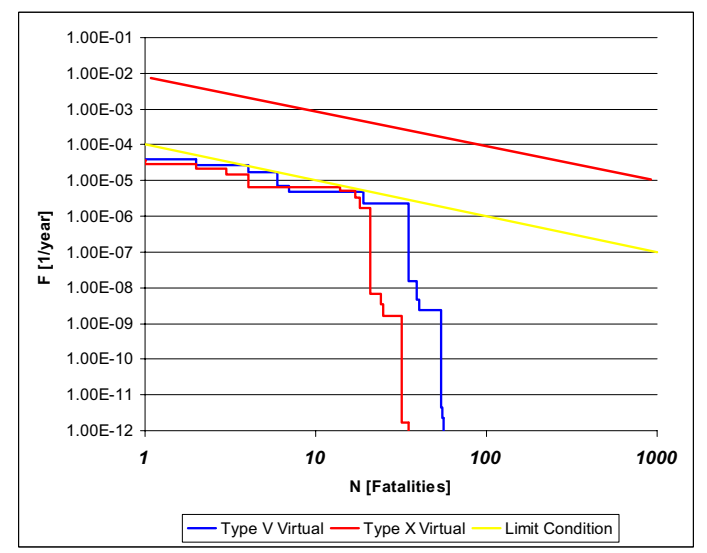

Figure 5: $\quad$ B.C.D. for V.T. of types V and X.

\section{Conclusive remarks}

The results of the risk analysis obtained according to the proposed procedure can be a decisional-making tool for the Administrative Authority (Article 4, European Directive 2004/54/CE). The European Directive as been completed and supplemented by an Italian Government Authorities act where appropriate criteria are included for both risk comparative analysis and an absolute acceptability limit and relative ALARP Zone. 


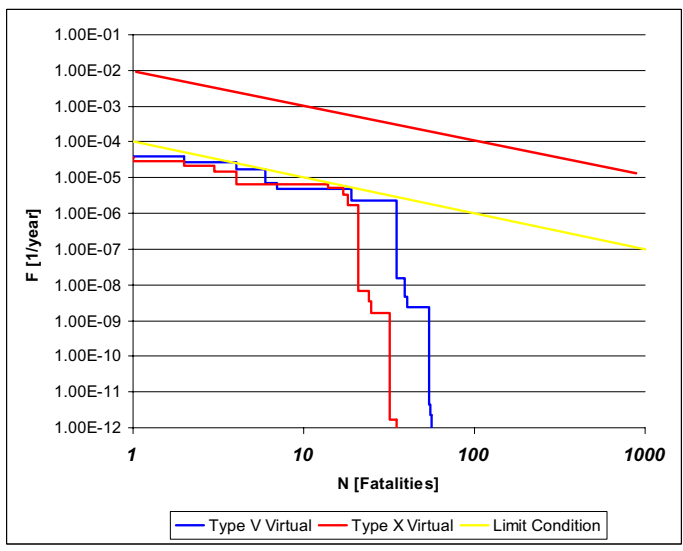

Figure 6: Virtual, Theoretical, Actual B.C.D. of the "Autostrade".

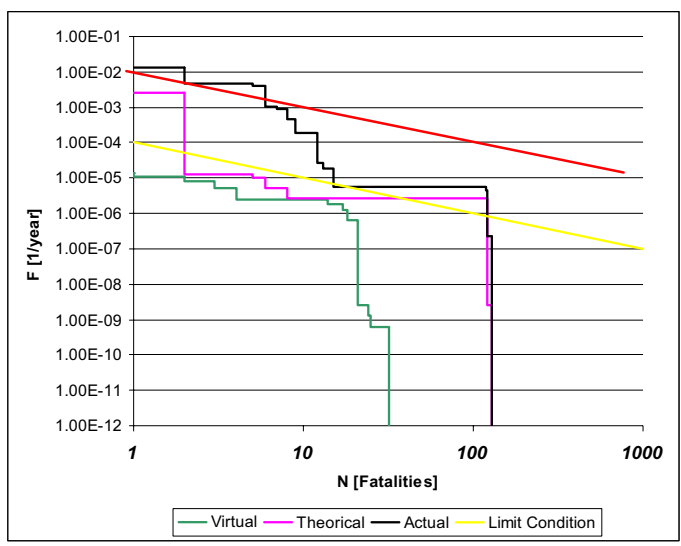

Figure 7: Virtual, Theoretical, Actual B.C.D. of the "ANAS 1".

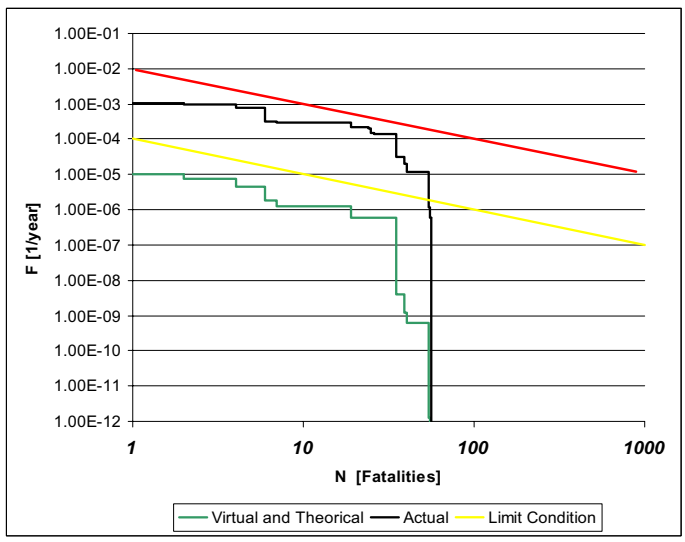

Figure 8: Virtual, Theoretical, Actual B.C.D. of the "ANAS 2". 
326 Safety and Security Engineering II

\section{References}

[1] Guarascio, M. et al., Risk Analysis and acceptability criteria, Malta, Safe, 2007

[2] EU Directive 54/2004/CE, On minimum safety requirements for tunnels in the trans-European road network

[3] D. Lgs. 05.06.2006, Road Tunnel Safety

[4] Risk Analysis for Road Tunnels, working group 2 management of road tunnel safety, PIARC, 2006

[5] Cafaro, E. et al., Metodo di progettazione della sicurezza nelle gallerie stradali, Torino, INTERTunnel 2006, convegno SIG, maggio 2006 